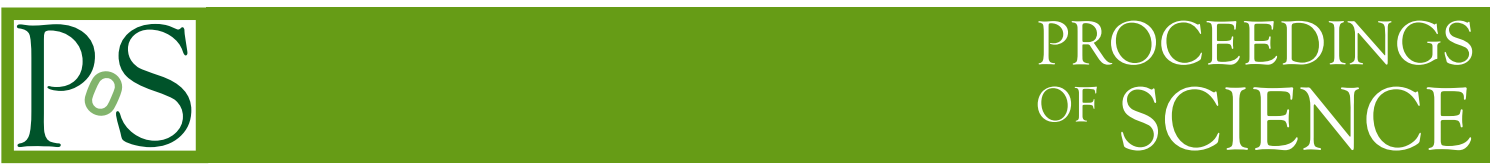

\title{
COMPTEL Reloaded: a heritage MeV data project
}

\author{
Werner Collmar \\ Max-Planck-Institut für extraterrestrische Physik, Garching, Germany \\ E-mail: wec@mpe.mpg.de

\section{Andrew Strong*} \\ Max-Planck-Institut für extraterrestrische Physik, Garching, Germany \\ E-mail: aws@mpe.mpg.de
}

The double-Compton telescope COMPTEL flew on the NASA Compton Gamma Ray Observatory (CGRO) satellite from 1991 to 2000, and is still the basis of most of our knowledge of the 1-30 MeV sky. Pending new missions like eAstrogam, for the next decade COMPTEL will still be a major resource for $\mathrm{MeV}$ gamma rays. A long-term effort to exploit heritage COMPTEL data is underway at MPE and MPA Garching. The full 9-year COMPTEL mission covered the entire sky. Several new developments are in progress for COMPTEL: the COMPTEL data analysis system was partly ported to Linux, new event processing techniques improve the background rejection, and new energy ranges are defined to avoid background lines. Time-of-flight background rejection has been improved using intra-detector resolution instead of just per detector, and this is combined with pulse-shape discrimination in a $2 \mathrm{D}$ analysis. A new source catalogue will be generated with the new event processing. The maximum-entropy skymapping method for COMPTEL has been updated to use current state-of-the art convolution on the sphere and the HealPix sky projection and the method has been adapted to modern hardware. New skymaps based on these developments are presented.

7th Fermi Symposium 2017

15-20 October 2017

Garmisch-Partenkirchen, Germany

\footnotetext{
*Speaker.
} 